\title{
Influence of calcium blockers on the SPR of erythrocytes
}

\author{
Olena V. Shynkarenko ${ }^{1}$, Orest Tril $^{2}$, Renata Wojnarowska ${ }^{3}$, Sergiy Prohorenko ${ }^{3,4}$ and E. M. Shergii ${ }^{3}$ \\ ${ }^{1}$ Lashkaryov Institute of Semiconductor Physics, NAS of Ukraine. Kyiv, Ukraine \\ ${ }^{1}$ Lviv State Oncology Regional Treatment and Diagnostic Centre. Lviv, Ukraine \\ ${ }^{3}$ Center for Microelectronics and Nanotechnology, University of Rzeszów. Rzeszów, Poland \\ ${ }^{4}$ Lviv Polytechnic National University. Lviv, Ukraine
}

\begin{abstract}
One of the promising areas of research is the impact of calcium channel blockers (CB) of biological fluids. This paper shows that the CB impact on a biological fluid can be efficiently combine with the surface plasmon resonance (SPR). It is shown that the addition of CB at the SPR measurements affect the stability of membranes and acts differently on the kinetics of erythrocytes ligament in the different groups of people.
\end{abstract}

\section{Introduction}

The structural and functional properties of red blood cells are mostly determined by rates of rigidity and elasticity of the membrane and by adhesive proteins on its surface [1]. High sensitivity of erythrocytes to the most of pathological changes in many diseases causes great diagnostic significance using a monitoring of the degree of elasticity of erythrocytes [2-4].

The main methods that are widely used in clinical practice to determine erythrocytes elasticity are method of filtering, micro-pipette method, method of centrifugation and others [4]. Nevertheless, they are quite labour-intensive and time-consuming measurements. For practical purposes it is important to have rapid research methods of red blood cell mass that use minimum quantities of biofluid samples. The ability to test the response of the red blood cell to the stress conditions (hypotonic or hypertonic solution, medicine etc.) is relevant. To solve these tasks the methods of diffractometric and the use of laser-traps $[5,6]$ are being developed. Unfortunately, they have important shortcomings: the method of laser-traps has a high error level of measurement $-19-41 \%[5,7]$. The present work proposes an effective method of the properties characterization of the red blood cells (RBC) using the surface plasmon resonance (SPR) measurements. We use calcium blockers [3] influence on the erythrocyte mass to demonstrate that SPR method can be effectively used to study effects of medicines on properties of the RBC.

\section{Experiment}

\subsection{Role of calcium blockers}

The widespread implementation of calcium blockers (CB) into clinical practice has led to a detailed study of calcium homeostasis [3].

It was found that ionized $\mathrm{Ca} 2+$ is involved in the regulation of the most of intracellular processes. Investigation of the $\mathrm{CB}$ influence on human erythrocytes makes it possible to obtain new fundamental knowledge concerning the organism as a whole and can be used in the future for diagnosing diseases. Properties of human erythrocytes are changed over time and are influenced by various factors.

To assess the stability of erythrocyte membranes, showing the condition of the organism as a whole, such methods as Definition of osmotic resistance of erythrocytes, Evaluation of peroxide resistance of erythrocytes, Determination of permeability parameters of the erythrocyte membranes using the method of urea hemolysis have been used [8-11]. These methods require considerable labor inputs and a long time to complete investigations. The SPR based sensors was used to perform the measurements in the current work. For above purpose highly specific proteins that bind complementary proteins from a liquid sample are immobilized on the working surface. On the basis of known investigated antigen-antibody pairs the vast majority of human blood tests for presence of antibodies to certain diseases are carried out by ELISA (EnzymeLinked ImmunoSorbent Assay) method. In the present work we propose a new method for assessing erythrocyte membranes using the SPR without the application of specific proteins.

\subsection{Description of the SPR experiment}

If a semitransparent $\mathrm{Au}$ film is placed at the interface (Fig.1), then under conditions of total internal reflection the SPR can occur. In this case, a polarized light beam is reflected on the interface of a noble metal surface

Corresponding author: o.shynkarenko@isp.kiev.ua

(C) The Authors, published by EDP Sciences. This is an open access article distributed under the terms of the Creative Commons Attribution License 4.0 (http://creativecommons.org/licenses/by/4.0/). 
vicinal dielectric layer at an angle superior to the total internal reflection angle.

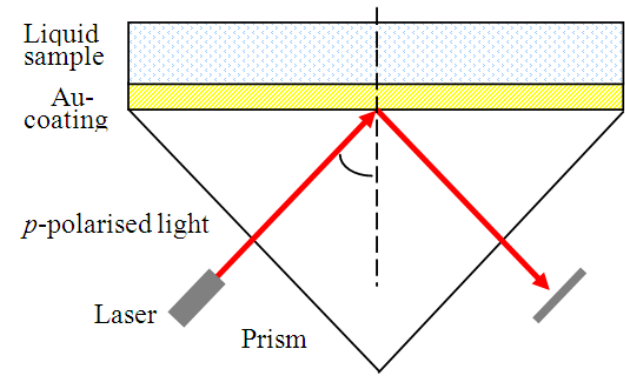

Fig. 1. Kretchmann prism coupling configuration for the SPR exitation.

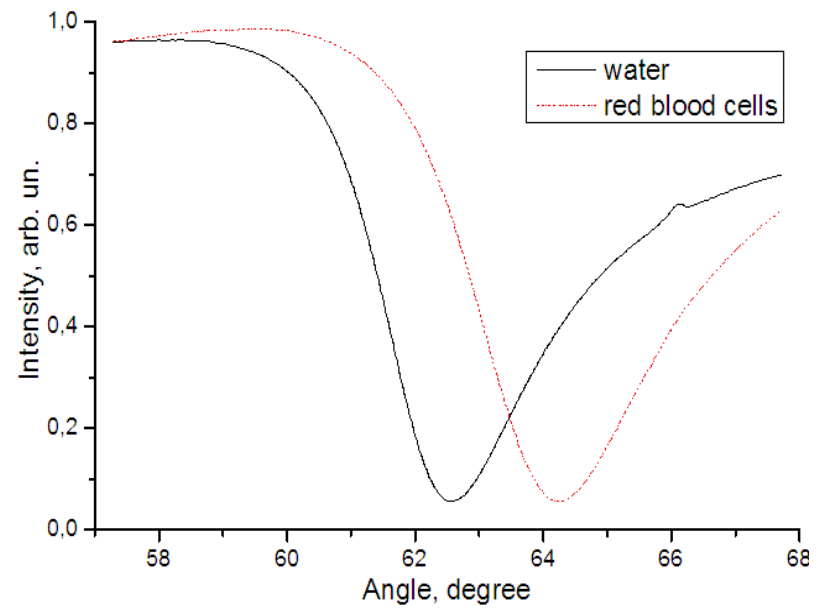

Fig. 2. Intensity of the reflected light versus angle of incidence for water and erythrocyte mass.

The underlying principle behind SPR is that a thin semitransparent layer of gold (or silver) will act as a mirror and reflect monochromatic p-polarised light projected at it except under special "matching" conditions. At the resonant angle the light energy will not be reflected, but rather converted into a collective oscillation of electrons in the metal film, generating the plasmon waveform. A direct plot of incident angle vs. reflected light intensity will show a marked loss of reflected energy at the resonant angle (Fig.2). The reflected light intensity will go sharply to almost zero at the resonant angle for a metal film of the right thickness.

\subsection{Erythrocytes adsorption to the surface of the SPR sensor}

The present paper devoted to study of the kinetics of erythrocytes adsorption to the surface of the SPR sensor. Resonance angle shift is proportional to the mass of the adsorbed film onto the surface of the sensor up to 200 $\mathrm{nm}$ in $\mathrm{Z}$ direction. Dimensions of erythrocytes are much more larger, approximately 2-7 microns, but under the influence of drugs affecting the stability of the membranes the erythrocytes change their properties that is displayed in the kinetic parameters of adsorption to the surface of the sensor. This can be conditioned by different types of packaging of erythrocytes near the surface as well as the erythrocytes membrane properties to change the shape or even just affect the kinetics of their interaction with the surface of the sensor.

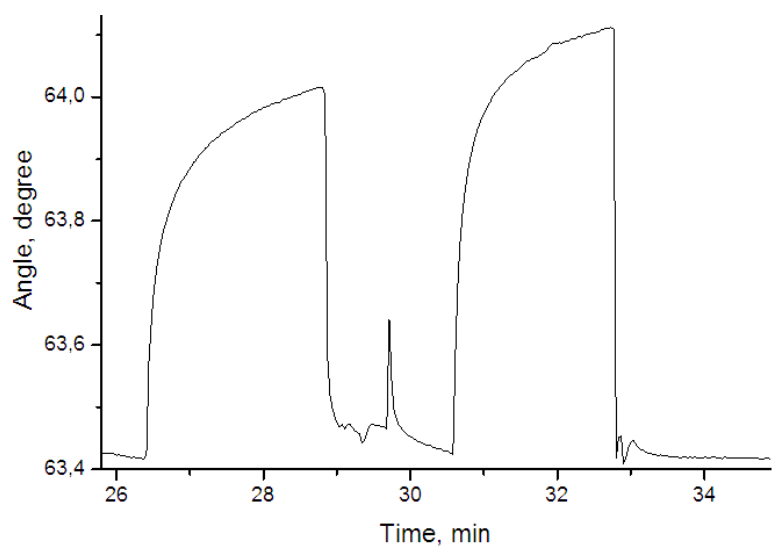

Fig. 3. Sensogram of SPR measurements for erythrocytes with addition of different $\mathrm{CB}$.

An example of different SPR kinetics are given in Figure 3. The samples of erythrocyte mass of human blood are used in the investigation. This method requires a minimum pre-processing of fresh venous blood samples and explores the red blood cell mass obtained after centrifugation of samples.

\section{Discussion and Conclusions}

As is shown above obtained results for different gropes of patients proposed method enables us to registered an observed real deference in position of minimum of reflection in the incident angle scale. That was confirmed on the 50 number grope of patient at clinical researches.

We have also demonstrated that the addition of $\mathrm{CB}$ that affect the stability of membranes acts differently on the kinetics of erythrocytes ligament in the different groups of people. To compare the results of different patients the difference of signals of erythrocytes kinetics in blood samples of patients without adding special agents and with the addition of drugs that is used. In this case the impact of differences in the density of blood for different patients is excluded. This method is numerical, simple, reproducible and requires a minimum of time 20-30 minutes for standardized methods, seemingly and it can be used to create a new methodology for determining the resistance of erythrocytes.

To determine the differences in the mechanism of interaction between erythrocytes under the influence of calcium channels the further research will be carried out.

The authors express their gratitude to employees of the Lviv State Cancer Center for providing blood samples for researches. 


\section{References}

1. J. Suart, C. S. Johnson, Baillibre's Clinical Haematology, I. 747 (1987)

2. R.M. Hochmuth, R.E. Waugh, Ann. Rev. Physiol., 49, 209-19, (1987)

3. M.R. Clark, A.C. Greenquist and S.B. Shohet, Blood, 48, 899 (1976)

4. C. Madigan and P. Malik, in book : Pathophysiology and Therapy for Haemoglobinopathies; Part I: Sickle Cell Disease, Vol. 8, (2006) pp. 1-23

5. T. Barnes, A. Shulman, A. Farone, M. Farone, D. Erenso, Optics and Photonics Journal, 3, 211 (2013)

6. M. Dao, C.T. Lim, S. Suresh, J. Mech. Phys. Solids. 51, 2259 (2003)

7. M.M. Brandao, A. Fontes, M. L. Barjas-Castro, L.C. Barbosa, F. F. Costa, C. L. Cesar, S. T. Saad, Eur. J. Haematol. 70, 207 (2003)

8. W. Grünberg, J. A. Mol, E. Teske. J of Vet. Int. Med.29,395 (2015)

9. J. O. Oyewale, J of Vet. Med. A 41.1, 62 (1994)

10. G. Del Mistro, S. Cervo, E. Mansutti, R. Spizzo, A. Colombatti, P. Belmonte, R Zucconelli, A. Steffan, V. Sergo, A. Bonifacio, Analitic. and Bioanalitic. Chem., 407, 3271 (2015)

11. M. Z. Vardaki, B. Gardner, N. Stone and P. Matousek,. Analyst, 140, 5112 (2015) 